should actually be continued.

An important step in studying signals in time-series, once a signal of interest has been identified in the power spectrum, is to generate the waveform in the time domain. This has already been done for the nominal 11-year wave occurring in many surface climate time-series. A similar waveform occurs in a 250 -year snow accumulation record from a northern mid-tropospheric site ${ }^{5}$. These waveforms, particularly the latter, exhibit pronounced amplitude modulation and phase shifts with respect to the sunspot cycle, which itself exhibits amplitude as well as frequency modulation. The waveforms derived from climate data may be approximately in phase with the solar cycle for a certain number of cycles only, before passing through a low-amplitude disordered sequence followed by a further group of clear cycles where the phase relationship may be retained or it may switch $180^{\circ}$ (ref. 4). With the exception of the latter effect, these observations may be substantially explained by the interaction of two atmospheric waveforms differing in period by about 1 to 2 years ${ }^{6}$. Thus, the solar cycle added to an approximately 10 year wave could produce the observed waveforms.

\section{National Hydrology Research Institute,} Saskatoon, Saskatchewan, Canada $57 N 3$ HS

1. Geiler, M.A. Nature 342, 15-16 (1989)

2. James, I.N. \& James, P.M. Nature 342, 53-55 (1989).

3. Tinsley, B.A. et al. J. geophys. Res. 94, 14783-14792 (1989)

. Currie, R.G. \& O'Brien, D.P. J. Clim. 8, 255-281 (1988)

5. Holdsworth, G. et al. Publ. 183, 71-79 (Int. Ass. hydrol Sci., 1989).

6. Castagnoli, C. et al. J. geophys. Res. 94, 11971-11976 (1989)

\section{Helical period of Z-DNA}

SIR-Pui Shing Ho of Oregon State University, and Michael Youngquist and Robert Driscoll of the California Institute of Technology, have independently pointed out that, in our paper "Scanning Tunnelling Microscopy of Z-DNA" (Nature 339, 484-486; 1989), the apparent helical period in our Figs 1 and 2 is considerably longer than the $43 \AA$ generally accepted for Z-DNA.

Both sets of authors have suggested that the $70 \times 46 \AA$ boxed area in Fig. $2 a$ of our paper (enlarged in Fig. 2b) may actually represent nearly two full turns of the helix. In that case, the prominent grooves would probably both be minor grooves corresponding to successive turns of the helix, since the bases in the major groove form a convex surface. This agrees with the observation by Ho that Fig. $1 b$ "shows ten peaks and nine valleys between 0 and 400 $\AA$ along the length of the fibre and is consistent with a $41.5-\AA$ spacing of each vertical displacement." Youngquist and Driscoll point out that "the molecular electrostatic potential surface has a major groove three times wider than the minor groove; the dark bands across the molecule in the STM image, however, are of nearly identical width", as would be the case for the van der Waals surface. They note that the 4-6- $\AA$ width of the dark bands accords well with the $3-5-\AA$ width obtained crystallographically. They also point out, correctly, that the Fourier transform frequencies at 20.4, 14.5 and $7 \AA$ in Fig. $1 c$ cannot necessarily be attributed to helical structural features, as they are close to harmonics of the $42-\AA$ period. Such harmonics would be expected since the helix is not a pure sinusoid.

We admit that these suggestions could well be the proper interpretation of our images. We did not notice the discrepancy in dimensions, and were seduced by the excellent visual match between the STM image and the electrostatic model (Fig. $2 c$ ). The van der Waals surface, while it gives reasonable correspondence with the grooves in Fig. $2 b$, does not show the variations in width perpendicular to the helix axis that are evident in Fig. $2 b, c$. On the other hand, if Fig. $2 b$ is scaled from 70 to $46 \AA$ and projected on Fig. $2 c$, the fit is remarkably good.

It seems likely that an STM image of DNA could reflect a complex mix of van der Waals and electronic properties. The interpretation of STM images of macromolecules such as DNA involves other issues in addition to the topographical contour, such as the size and structure of the probe tip, its response as it scans along graphite and approaches a thick molecule from the side, its pressure on the sample, and the inhomogenous electronic structure of the polymer. The most dependable information lies in the periodicities, those features which repeat regularly, regardless of the mechanism of image formation. Judged by this criterion, our images clearly show the left-handed helix and helix-repeat characteristics of Z-DNA.

PATRICIA G. ARSCOTT

GIL LEE

VICTORA. BLOOMFIELD D. FenNeLl Evans

Department of Biochemistry,

University of Minnesota,

St Paul, Minnesota 55108, USA

and

Center for Interfacial Engineering, University of Minnesota,

Minneapolis, Minnesota 55455, USA

\title{
Nepotism in the honey bee
}

SIR-Reports that honey-bee (Apis mellifera) workers discriminate nepotistically among nestmate patrilines, by Page et al. ${ }^{1}$ and others (for example, refs 2-8), have generated considerable interest. But Queller et al. ${ }^{9}$ recently found that Polistes annularis wasps, under natural conditions, do not preferentially join more closely related natal nestmates during foundation of their spring colonies. Similarly, within colonies of the polygynous carpenter ant Camponotus planatus, workers fail to distinguish their mother queen, sister workers or sister virgin queens from those of other naturally cohabiting nestmate matrilines (N.F.C. and $\mathrm{S}$. Cover, unpublished results). The discrepancy between the results obtained in wasps and ants and in the honey bee could reflect a unique kin-recognition ability of the latter. Alternatively, we suggest that the composition of experimental colonies containing artificially low numbers of patrilines, of artificially high phenotypic distinctiveness, may yield nepotism as an artefact ${ }^{10}$

Apparent kin recognition can easily arise as the accidental by-product of different discrimination mechanisms based on genetically correlated cues (such as species or mate recognition $)^{11}$. Social insects are acutely sensitive to foreign odours normally used to identify and reject non-nestmates from the colony. Honey-bee workers in colonies of artificial phenotypic heterogeneity may per- ceive their peculiar nestmates as smelling more like foreigners than like ordinary nestmates. Discrimination in such behaviours as worker interactions, swarming and queen brood rearing may result, not from a preference for one's own patriline, but from a bias against the other, though not reaching the threshold necessary for rejection ${ }^{7.10}$. Strange-smelling larvae may in addition elicit discrimination that is normally directed against diseased brood, though again below the threshold for removal; such behaviour is known to be important for disease resistance in honey bees ${ }^{12}$

Various experimental methods have been used to form genetically mixed colonies of honey bees, all of which combine a diversity of phenotypes which may not typically coexist. In early studies, colonies containing multiple kin groups were formed by adopting brood between foreign nests, of known or assumed relatedness ${ }^{2.3}$. An apparent improvement was introduced by artificially inseminating a queen with drone sperm bearing heritable colour markers, yielding half-sibships visually distinguishable to the investigators (for example, refs 4-8). Because bees recognize relatives by olfaction, the colours themselves were usually assumed not to affect recognition responses. But marker genotypes have been shown to alter the bees' odour phenotypes as well. When a queen heterozygous for a recessive marker is inseminated with sperm 